\title{
Radial Artery Pseudoaneurysm Treated with a Compression Bandage after Invasive Blood Pressure Monitoring in a Patient with a Traumatic Injury
}

\author{
Seong Pyo Mun, M.D., Yoo Seok Kim, M.D., Nam Kyu Choi, M.D., Sung Soo Kim, M.D., and Young Sun Yoo, M.D. \\ Department of Surgery, Chosun University School of Medicine, Gwangju, Korea
}

Dear Editor:

We report a case of radial artery pseudoaneurysm (RAP) in the wrist due to catheterization that was treated with a compression bandage.

Trans-radial access (TRA) is increasingly being used for diagnostic coronary angiography, percutaneous coronary intervention (PCI), and invasive arterial pressure monitoring. It is preferred to traditional femoral artery access, because it is associated with significantly fewer access site complications.[1] It is very important to know the complications associated with this access technique and how to prevent and manage them. Some of the common complications of TRA include asymptomatic radial artery occlusion, non-occlusive radial artery injury, and radial artery spasm. Symptomatic radial arterial occlusion, pseudoaneurysm, and radial artery perforation are rarely reported. Generally, access site complications can be minimized by avoiding multiple punctures, selecting smaller sheaths, performing gentle catheter manipulation, providing adequate anticoagulation therapy, using appropriate compression devices, and avoiding prolonged high-pressure compression. Additionally, the careful observation of pain, numbness, and hematoma formation during the immediate post-procedure period is essential for preventing catastrophic hand ischemia.[2] In contrast to TRA for coronary intervention, a 22- to 24-Gauge (G) angiocatheter without a sheath is used for TRA for invasive monitoring of arterial blood pressure. Radial artery pseudoaneurysm (RAP) for invasive monitoring is an extremely rare complication associated with TRA. We describe a case of RAP that was successfully treated using a compression bandage. We believe that the safety, efficacy, and minimal invasiveness of this method make it feasible for use as a treatment in selected patients with RAP.

A 56-year-old man was referred to our hospital after sustaining a blunt abdominal injury in a motor vehicle crash. Because the patient had unstable vital signs, we performed exploratory laparotomy, which showed hemoperitoneum and active

\section{Received on May 13, 2016 Revised on August 10, 2016 Accepted on August 11, 2016 \\ Young Sun Yoo \\ Department of Surgery, Chosun University School of Medicine, 365 Pilmun-daero, Donggu, Gwangju 61453, Korea \\ Tel: $+82-62-220-3060$ \\ Fax: +82-62-228-3441 \\ E-mail: ysyoo@chosun.ac.kr}

*No potential conflict of interest relevant to this article was reported. 
bleeding from the pancreaticoduodenal artery. The bleeding vessel was ligated, and there was no evidence of any associated injuries to the surrounding organs.

Radial arterial catheterization was carried out by anesthesiologist to monitor the blood pressure during the operation. A 22-G angiocatheter was inserted without any problem on the first trial. The arterial wave was showed well and blood sampling could be performed without resistance. It was used for invasive hemodynamic monitoring and blood sampling in the trauma intensive care unit after operation. The catheter was removed on the sixth postoperative day, because the patient's condition improved and invasive monitoring was unnecessary. Simple manual compression was performed for $10 \mathrm{~min}$ utes. We did not use anticoagulation therapy. The patient did not have any complaints for next couple of days. On the tenth postoperative day, a painful pulsatile mass with redness was noted at the radial aspect of the right wrist
(Fig. 1). Ultrasonography findings showed an echoic mass measuring $11 \times 8 \times 7 \mathrm{~mm}$, and findings of color Doppler imaging showed a swirling pattern of internal flow consistent with RAP (Fig. 2). Results of Allen's test

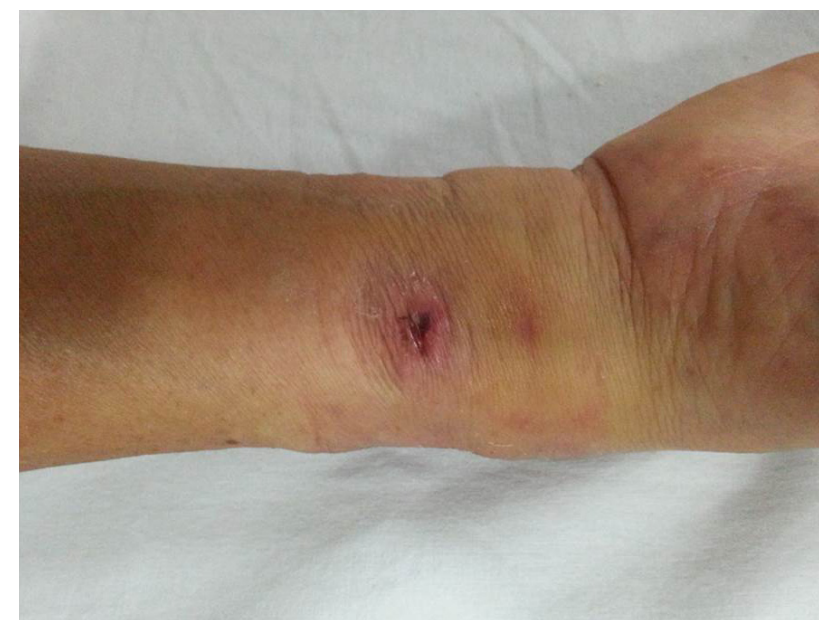

Fig. 1. Photograph of the right wrist. Painful, erythematous swelling was observed at the site of radial artery catheterization.
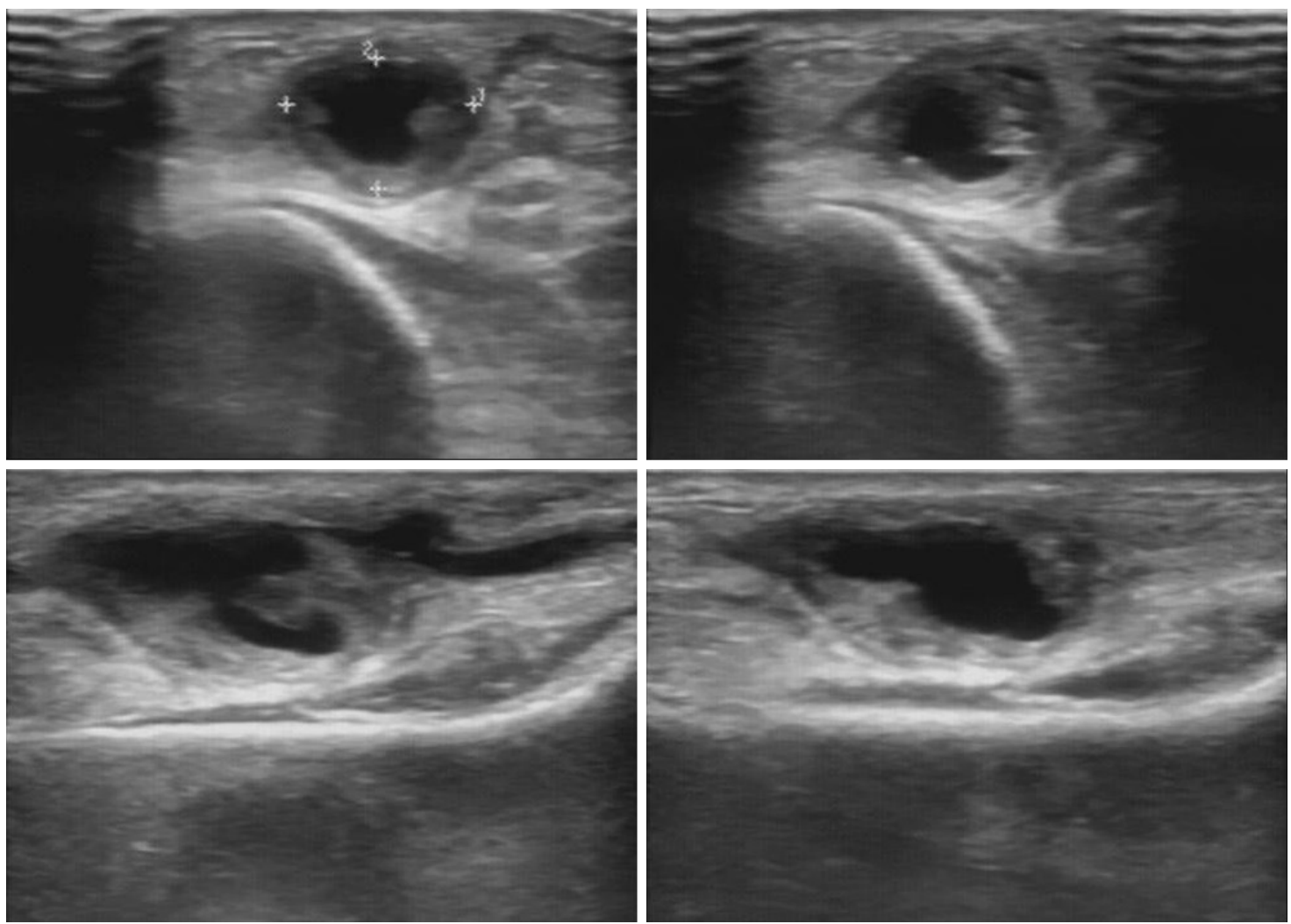

Fig. 2. Ultrasonography of the right wrist demonstrating an echoic mass measuring $11 \times 8 \times 7 \mathrm{~mm}$ and a swirling pattern of internal flow consistent with pseudoaneurysm of the radial artery. 
indicated normal circulation in the right hand. Considering the shape and size of the RAP, Hemostop (Kyowa Hakko Kirin Co., Ltd., Seoul, Korea) was applied. This comprises a small oval pad that compresses the insertion site of the catheter and an elastic band that wraps around the wrist and supports the pad (Fig. 3). The bandage was used originally for hemostasis after hemodialysis. Clinical and Doppler ultrasound reassessment after 1 week showed a decreased pseudoaneurysm size and thrombus formation. Digital blood flow and digital blood pressure were preserved, and there was no ischemic change in the hand and fingers (Fig. 4). Compression was maintained for one more week, and thrombotic occlusion of the RAP was shown on follow-up sonography. The finger-brachial indexes indicated a normal digital blood pressure (Fig. 5). After 18 months of follow-up, the patient remains symptom-free with normal circulation of the hand.

The radial artery is regarded as the best arterial catheterization site because it has favorable anatomic relationships with surrounding structures; there are no major nerves and veins, which makes it desirable for PCI and arterial catheterization for blood pressure monitoring.
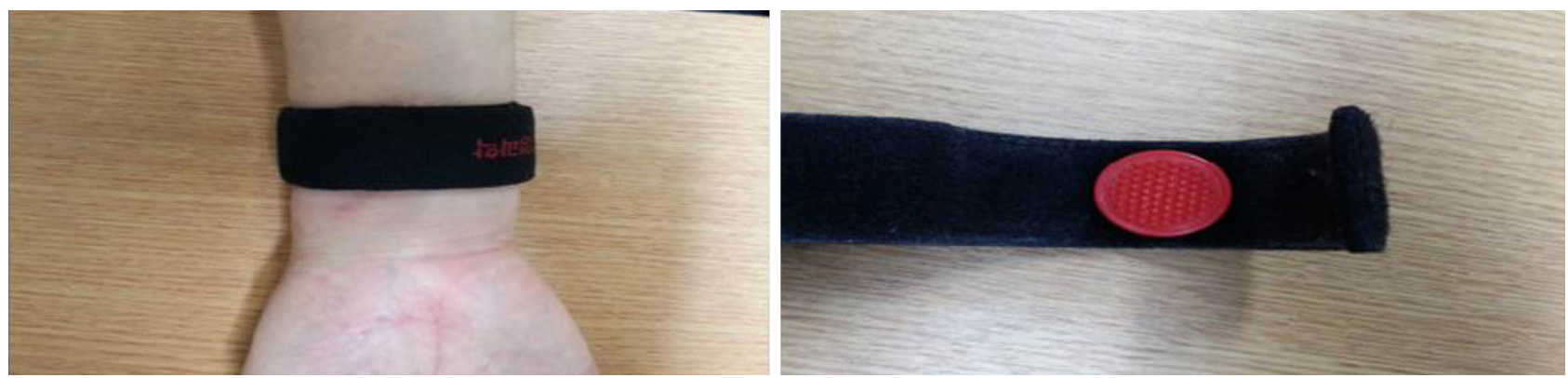

Fig. 3. Photograph of the compression band. The band was comprised of an oval pad for compression and an elastic bandage to wrap the wrist.
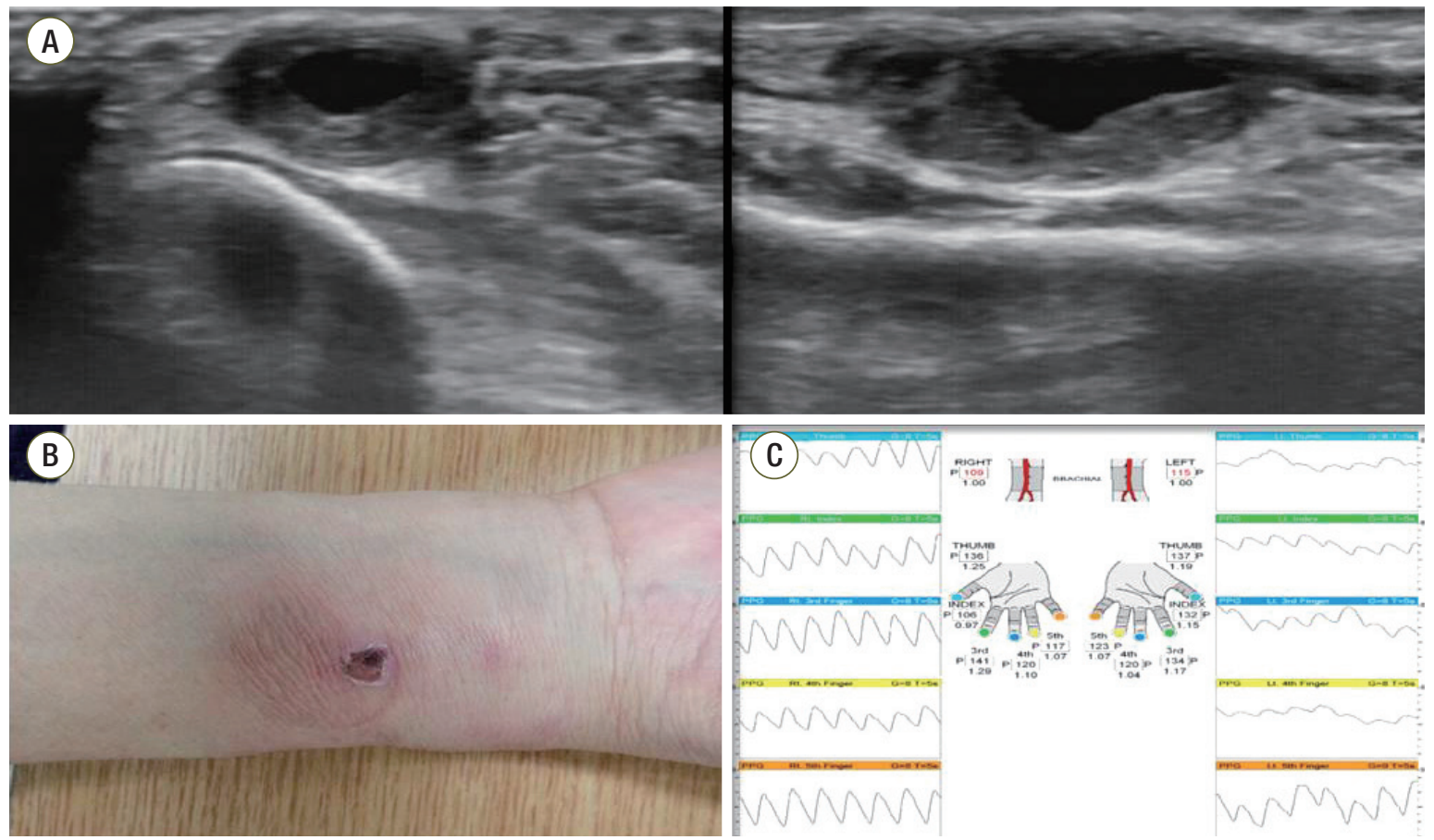

Fig. 4. (A) Ultrasonography of the wrist. (B) Photograph of the right wrist. (C) Finger-brachial indexes of both hands. A Doppler ultrasonogram 1 week later revealed a decreased pseudoaneurysm size and thrombus formation. The finger-brachial indexes indicated normal digital blood pressure. 

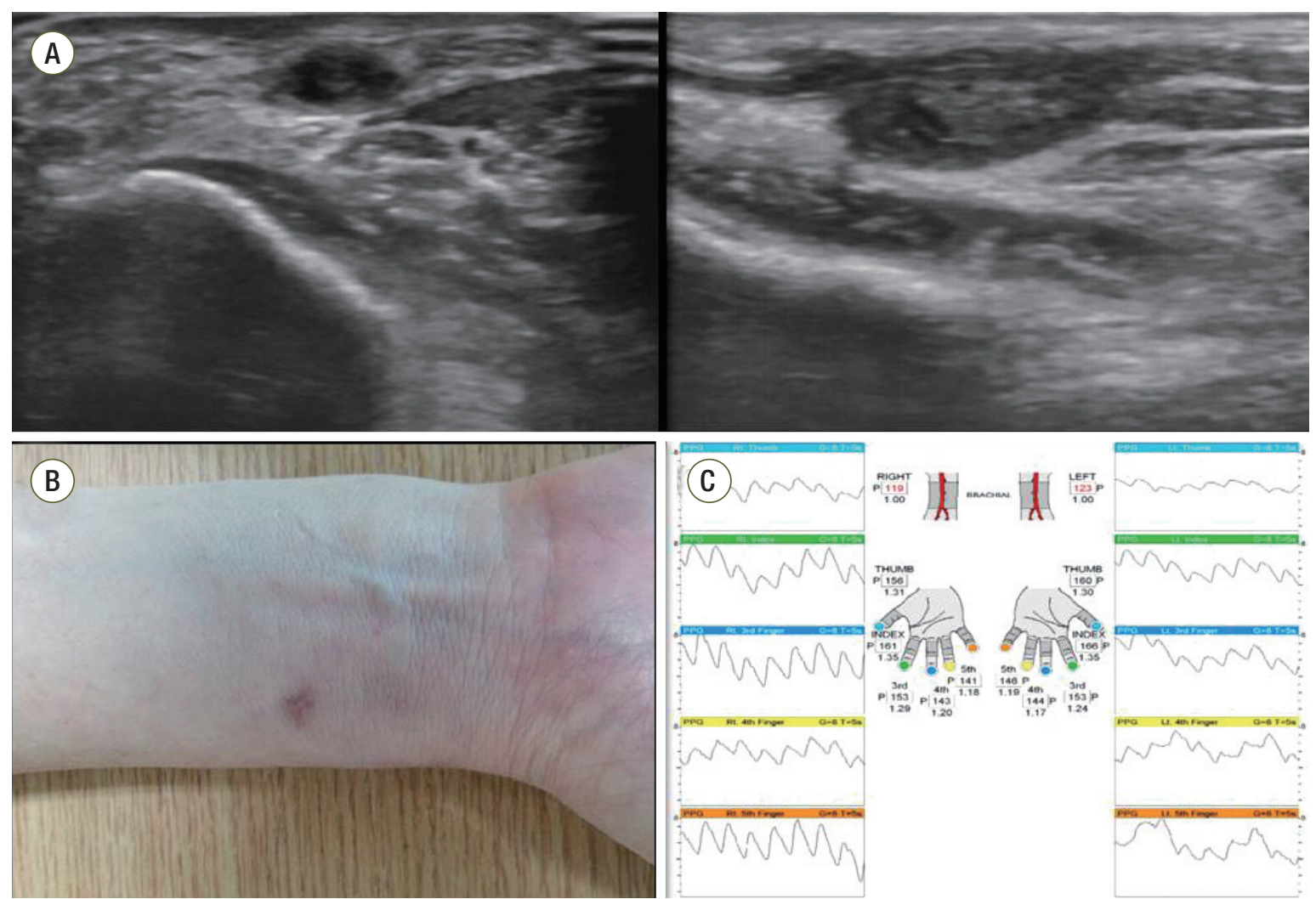

Fig. 5. (A) Ultrasonography of the wrist. (B) Photograph of the right wrist. (C) Finger-brachial indexes of both hands. A Doppler ultrasonogram obtained 2 weeks later revealed thrombotic occlusion of the radial artery pseudoaneurysm. The finger-brachial indexes indicated normal digital blood pressure.

Compared to the femoral access, TRA is associated with decreased vascular complications. A randomized study showed that TRA is associated with a lower complication rate, higher quality of life, and lower cost. $[1,3,4]$ In contrast to coronary intervention using a 5-French sheath, radial arterial catheterization for invasive blood monitoring does not require a sheath. The angiocatheter is inserted directly into the radial artery. Because the angiocatheter is very thin, RAP is a very rare complication after invasive blood monitoring. Generally, access site complications are associated with multiple punctures, larger sheaths, rough catheter manipulation, inadequate anticoagulation therapy, the use of inappropriate compression devices, and prolonged high-pressure compression. Although there is no technical error associated with catheterization, RAP is a possible complication if there is an infection at the access site. The usual causative organisms are Staphylococcus and fungi.[5]
The diagnosis is easily made by using duplex sonography, as it shows the aneurysmal sac, thrombosis in the pseudoaneurysm, and turbulent flow.[6] It is important to know the shape, size, and relationship to the radial artery to select the best management approach.

There are several options for treating RAP: a compressive bandage, ultrasound-guided thrombin injection, and microsurgery.

A compressive bandage is a simple, noninvasive, and cost-effective procedure that is the first-line treatment for small and uncomplicated RAP. Compression management is initially applied to the pseudoaneurysm of the arteriovenous fistula sites for hemostasis. Commercial compression devices are used for hemostasis after radial artery catheterization because of convenience.[7,8] In contrast with PCI, invasive blood pressure monitoring only requires a thin intra-arterial catheter rather than an angiographic sheath with a large diameter. A simple elas- 
tic bandage with a compression pad seems effective for managing RAP caused by radial arterial catheterization. The problem is determining how long surgeons should compress the TRA site. The longer surgeons compress the TRA site, the higher the incidence of radial arterial occlusion. Unfortunately, there is no definitive guideline for the duration of compression for RAP. It would be better to use ultrasound findings and the patient's symptoms to determine the optimal compression duration. If compression is applied, there is a chance of causing ischemic injury of the hand due to radial arterial occlusion if the ulnar arterial patency is not good. Allen's test should be performed to ensure patency of the ulnar artery.

An ultrasound-guided thrombin injection can be used to manage RAP. Since it can cause distal thrombotic occlusion, it should be used in saccular-type aneurysms with a neck. The thrombin should be injected directly into the RAP. After a study reported success with treating RAP in a patient with Behcet's syndrome, many trials have studied the use of a thrombin injection for treating RAP. Pseudoaneurysmal sacs were usually occluded with a thrombus after a single injection. Follow-up Doppler ultrasound showed that nearly all cases were a procedural success, and the complication rate was very low. If RAP is a saccular-type aneurysm with a neck, a thrombin injection into the RAP is a safe treatment option.[9]

Surgical repair is rarely indicated, because nonoperative management is usually successful. The most common surgical procedure is ligation of the radial artery if the ulnar artery is intact. End-to-end anastomosis or bypass is also a possible surgical option. However, as endto-end anastomosis and bypass are associated with surgical difficulty, they should be used when non-invasive management or ligation of the radial artery cannot be performed.

It is difficult to determine when RAP occurred in this patient, but inadequate manual compression of the access site seems to be the cause. An infection is also a possible cause of RAP because there was erythematous swelling around the access site. Since we did not perform the physical examination carefully, the diagnosis of RAP was delayed. Because the RAP was saccular shape without neck and ulnar artery was intact, simple compression was applied and successful. A commercial compression device seems to be a substitute not only for a manual compression of access site of invasive blood pressure monitoring after catheter removal but also for the management of RAP. Most importantly, Allen's test should be performed always before radial arterial catheterization in cases in which radial arterial occlusion is an unavoidable complication.

\section{Acknowledgements}

The present study was supported by grants from Chosun University Hospital 2016.

\section{ORCID}

Seong Pyo Mun http://orcid.org/0000-0002-7067-4751

Young Sun Yoo http://orcid.org/0000-0002-0451-9788

\section{References}

1) Klinke WP, Hilton JD, Warburton RN, Warburton WP, Tan RP: Comparison of treatment outcomes in patients $>$ or $=80$ years undergoing transradial versus transfemoral coronary intervention. Am J Cardiol 2004; 93: 1282-5.

2) Bazemore E, Mann JT 3rd: Problems and complications of the transradial approach for coronary interventions: a review. J Invasive Cardiol 2005; 17: 156-9.

3) Yang YJ, Kandzari DE, Gao Z, Xu B, Chen JL, Qiao $\mathrm{SB}$, et al: Transradial versus transfemoral method of percutaneous coronary revascularization for unprotected left main coronary artery disease: comparison of procedural and late-term outcomes. JACC Cardiovasc Interv 2010; 3 : 1035-42.

4) Kajiya T, Agahari F, Wai KL, Tai BC, Lee CH, Chan $\mathrm{KH}$, et al: A single-center experience of transitioning from a routine transfemoral to a transradial interven- 
tion approach in ST-elevation myocardial infarction: impact on door-to-balloon time and clinical outcomes. J Cardiol 2013; 62: 12-7.

5) Stansby G, Smout J, Chalmers R, Lintott R: MRSA infected pseudoaneurysms of the radial artery. Surgeon 2003; 1: 108-10.

6) Uhlemann M, Möbius-Winkler S, Mende M, Eitel I, Fuernau G, Sandri M, et al: The Leipzig prospective vascular ultrasound registry in radial artery catheterization: impact of sheath size on vascular complications. JACC Cardiovasc Interv 2012; 5: 36-43.

7) Liou M, Tung F, Kanei Y, Kwan T: Treatment of ra- dial artery pseudoaneurysm using a novel compression device. J Invasive Cardiol 2010; 22: 293-5.

8) Bhat T, Teli S, Bhat H, Akhtar M, Meghani M, Lafferty J, et al: Access-site complications and their management during transradial cardiac catheterization. Expert Rev Cardiovasc Ther 2012; 10: 627-34.

9) Reus M, Vázquez V, Alonso J, Morales D, Rodríguez JM: Treatment of a radial artery pseudoaneurysm with ultrasound-guided percutaneous thrombin injection in a patient with Behcet's syndrome. J Clin Ultrasound 2003; 31: 440-4. 